Canad. J. Math. Vol. 52 (2), 2000 pp. 225-247

\title{
Localization in Categories of Complexes and Unbounded Resolutions
}

\author{
Leovigildo Alonso Tarrío, Ana Jeremías López and María José Souto Salorio
}

\begin{abstract}
In this paper we show that for a Grothendieck category $\mathcal{A}$ and a complex $E$ in $\mathrm{C}(\mathcal{A})$ there is an associated localization endofunctor $\ell$ in $\mathbf{D}(\mathcal{A})$. This means that $\ell$ is idempotent (in a natural way) and that the objects that go to 0 by $\ell$ are those of the smallest localizing (= triangulated and stable for coproducts) subcategory of $\mathbf{D}(\mathcal{A})$ that contains $E$. As applications, we construct $\mathrm{K}$-injective resolutions for complexes of objects of $\mathcal{A}$ and derive Brown representability for $\mathbf{D}(\mathcal{A})$ from the known result for $\mathbf{D}(R$-mod $)$, where $R$ is a ring with unit.
\end{abstract}

\section{Introduction}

One of the key results in stable homotopy theory is Bousfield's localization, namely for a homology theory $E$, there is an associated localization functor, i.e., an idempotent functor which sends every spectrum to a $E$-local one, universally ([Bo1], [Bo2]). This can be conveniently expressed in the language of triangulated categories. Let HoSp denote the stable homotopy category of spectra and identify $E$ with the spectrum that represents it. The theorem says we have an equivalence between the full subcategory of $E$-local objects and the quotient subcategory of $H o S p$ by the localizing subcategory generated by $E$.

It has been clear for some time now that it is very fruitful to transport the results from stable homotopy to homological algebra exploiting the similar structure that HoSp and derived categories share, they are triangulated categories with a "model", the categories of CW-spectra and of complexes, respectively. It is also useful to study the problem of localization in a derived category of sheaves over a space, because it gives information about the geometry expressed by the initial category of sheaves. For instance, Neeman (see [N2]) has shown a relation between localizations in $\mathbf{D}(R)$ and subsets of $\operatorname{Spec}(R)$.

In this paper we show that the analogous localization theorem to Bousfield's holds for the derived category of an Abelian Grothendieck category $\mathcal{A}$. We start with an interpretation of Bousfield's kind of arguments in the derived category of a ring and then deduce the theorem for $\mathbf{D}(\mathcal{A})$. Most of the interesting situations that arise in practice are categories of this kind. We work in the unbounded derived category because our arguments rely in existence of arbitrary coproducts. As an application, we get unbounded injective resolutions in this generality. To the best of our knowledge there is no published proof of this fact.

The first result of the existence of this kind of resolutions is Spaltenstein's [Sp], who works in the derived category of sheaves over a topological space. A short and elegant proof in the derived category of modules over a ring was given by Bökstedt and Neeman

Received by the editors May 7, 1998; revised December 3, 1998.

All three authors partially supported by Xunta de Galicia through research project XUGA20701A96 and Spain's DGESIC grant PB97-0530.

AMS subject classification: Primary: 18E30; secondary: 18E15, $18 \mathrm{E} 35$.

(c) Canadian Mathematical Society 2000. 
$[\mathrm{BN}]$. This proof was later used in some contexts where arbitrary (or at least countable) products are exact, see $[\mathrm{K}]$ and $[\mathrm{CtHK}$, appendix], but this assumption seldom holds in categories of sheaves of modules. We have been informed by F. Morel that he also has a proof of the result using Quillen's model categories. Franke sent us the preprint [F] after the localization theorem was obtained. In it, the existence of unbounded resolutions is deduced from Brown representability. In our approach, Brown representability is obtained as a consequence of localization.

Our proof uses some ideas of Neeman. In the paper [AJL, p. 2, footnote 1], it is asserted one can get a simple proof of the fact using Gabriel-Popescu embedding theorem. The subtle point is to extend the inclusion functor $\mathcal{A} \rightarrow R$-mod to derived categories. This follows from the localization theorem (see Corollary 5.2).

We have tried to make the paper self-contained modulo standard facts about derived categories. However, a reader just wanting to see a proof of the existence of K-injective resolutions for complexes of objects in a Grothendieck category, could opt for reading the statement of Proposition 4.5 and follow the next section up to Theorem 5.4. Alternatively, a reader familiar with the Brown representability theorem of [N3] could follow an idea of Neeman: read from the beginning of Section 5, give an alternative proof of Corollary 5.2 as suggested in the remark after it and then continue again up to Theorem 5.4.

Next, we describe in some detail the contents of the paper. The first section is preliminary and just sets up the general concepts of localization that are going to be used and mostly well-known remarks. The reader is advised to skip it. In the second, we develop a theory of homotopy limits for complexes. It may well agree with the simplicial one developed by Bousfield and Kan [BK] using the Dold-Kan correspondence [W, (8.4)], but we are just doing here an exposition tailored to our needs. This also helps to streamline the prerequisites. The key observation is Theorem 2.2 which establishes the agreement of the usual limit of complexes with our homotopy construction.

The third section is devoted to proving Theorem 3.1 which states that the homotopical direct limit of a family of complexes in a localizing subcategory $\mathcal{L}$ of $\mathbf{D}(\mathcal{A})$ belongs to $\mathcal{L}$. The proof goes by exhibiting a countable filtration of the homotopical direct limit and is somehow inspired by the correction of Bousfield's argument in [Bo2a].

In the next section we start recalling that if the generator of $\mathcal{A}$ is projective then unbounded projective resolutions exist in $\mathbf{K}(\mathcal{A})$, which includes the case $\mathcal{A}=R$-mod. With these tools, we are able to prove the localization theorem for the derived category of $R$ modules (Proposition 4.5): a localizing subcategory generated by a set in $\mathbf{D}(R)$ defines a localization functor.

Finally, in Section 5, we prove the theorem of existence of localization in the context of derived categories (Theorem 5.7). We deduce it from the previous one, but we have had to follow a somehow indirect path. We show that the Gabriel-Popescu embedding can be extended to the derived category, showing that the derived category of a Grothendieck category is a localization of the derived category of the category of modules over a ring. Then, we show the existence of K-injective resolutions for complexes on a Grothendieck category using the previous fact and the usual explicit construction for complexes of modules over a ring (see $[\mathrm{BN}]$ and $[\mathrm{Sp}]$ ). This allows us to prove the theorem of existence of localizations in $\mathbf{D}(\mathcal{A})$. We give some examples that relate our theory to localizations in Abelian categories and show that there are localizations in $\mathbf{D}(\mathcal{A})$ that do not come from localizations in $\mathcal{A}$. The section ends with a proof of Brown representability, namely, every contravari- 
ant homological functor from $\mathbf{D}(\mathcal{A})$ that takes coproducts to products is representable. In the proof we use that Brown representability holds for $\mathbf{D}(R)$ which was proved by Neeman in $[\mathrm{N} 3]$.

In our research we have benefited from conversations and hints by several people. Amnon Neeman helped us a lot, specially sharing with us his ideas about K-injective resolutions and his criticisms about a first version of this paper. J. L. Gómez Pardo helped us to reach the right track about set-theoretic puzzlements. We thank Joe Lipman for his interest and encouragement. We also benefited from remarks by Jeff Smith, Bernhard Keller, and from several other people who attended our lectures while this work was being developed.

\section{Preliminaries}

Let $\mathbf{T}$ be a triangulated category. Following Adams and Bousfield, we define a localization in $\mathbf{T}$ as a pair $(\ell, \eta)$ where $\ell: \mathbf{T} \rightarrow \mathbf{T}$ is a triangulated endofunctor, and $\eta: 1 \rightarrow \ell$ a natural transformation such that $\ell \eta_{X}=\eta_{\ell X}$ for any $X$ in $\mathbf{T}$ and $\eta$ induces an isomorphism, $\ell X \cong$ $\ell \ell X$. We will make the usual abuse of notation and refer to $\ell$ as a localization functor in $\mathrm{T}$, leaving implicit the natural transformation $\eta$. Such a localization functor determines a subcategory of $\mathbf{T}$, the (strictly) full subcategory whose objects are those $X$ such that $\ell X=0$. The main problem we want to address is to what extent does this subcategory determine $\ell$. To make this article reasonably self-contained, we will recall in this section some wellknown facts with mere sketches of proofs.

Lemma 1.1 Let $X$ be an object of $\mathrm{T}$, then $\ell X=0$ if, and only if, $\operatorname{Hom}(X, \ell Y)=0$, for every $Y$ in $\mathbf{T}$.

Proof If $\ell X=0$, it is enough to realize that any map $X \rightarrow \ell Y \cong \ell \ell Y$ factors trough $\ell X$. Conversely, the canonical map $\ell X \rightarrow \ell \ell X$ is 0 and an isomorphism, therefore $\ell X=0$.

Proposition 1.2 The full subcategory $\mathcal{L} \subset \mathrm{T}$ whose objects are those $X$ such that $\ell X=0$ is triangulated and stable for coproducts, that is, whenever a coproduct of a family of such objects exists in $\mathbf{T}$ is an object of $\mathcal{L}$.

Proof It is clearly triangulated because $\ell$ is a triangulated functor. Now, for every $Y$ in $\mathbf{T}$, $\operatorname{Hom}\left(\bigoplus X_{i}, \ell Y\right)=\prod \operatorname{Hom}\left(X_{i}, \ell Y\right)$; therefore if $\ell X_{i}=0$ for every $i$, then $\ell\left(\bigoplus X_{i}\right)=0$.

Remark Suppose $\mathbf{T}$ has coproducts. If a triangulated subcategory $\mathcal{L}$ of $\mathbf{T}$ is closed for coproducts of $\mathbf{T}$, it is also closed for direct summands, we mean that if $X \in \mathcal{L}$ and $X \cong$ $X_{1} \oplus X_{2}$ in $\mathbf{T}$, then also $X_{1}$ and $X_{2}$ belong to $\mathcal{L}$ as follows from Eilenberg's swindle ${ }^{1}$. But this characterizes thick subcategories (Rickard's criterion: see [Ri, Proposition 1.3]) and by Verdier's theory ([Ve]), the quotient category $\mathbf{T} / \mathcal{L}$ is defined. Recall that the elements in $\operatorname{Hom}_{\mathbf{T} / \mathcal{L}}(X, Y)$ are diagrams $X \stackrel{s}{\leftarrow} X^{\prime} \stackrel{f}{\rightarrow} Y$ such that the vertex of the triangle with base $s$ is in $\mathcal{L}$. A map like $s$ will be called a $\mathcal{L}$-quasi-isomorphism.

\footnotetext{
${ }^{1} X_{1}$ can be identified with the cone of the map $\bigoplus_{\mathbb{N}} X \rightarrow \bigoplus_{\mathbb{N}} X$ defined as the canonical projection $X_{1} \oplus$ $\left(X_{2} \oplus X_{1} \oplus X_{2} \oplus \cdots\right) \rightarrow X_{2} \oplus X_{1} \oplus X_{2} \oplus X_{1} \oplus \cdots$
} 
Definition A triangulated subcategory $\mathcal{L}$ of $\mathbf{T}$ closed for coproducts will be called a $10-$ calizing subcategory. The full subcategory of $\mathbf{T}$ whose objects $X$ are $\mathcal{L}$-local, i.e. such that $\operatorname{Hom}(Y, X)=0$ for every $Y$ in $\mathcal{L}$, will be denoted by $\mathcal{L}^{\perp}$.

If $\ell$ is a localization functor, the full subcategory $\mathcal{L} \subset \mathbf{T}$ whose objects are those $X$ such that $\ell X=0$ is a localizing subcategory. We will call $\mathcal{L}$ the localizing subcategory associated to $\ell$. As a consequence of Lemma $1.1, \mathcal{L}^{\perp}$ agrees with the essential image of $\ell$.

Lemma 1.3 If $\mathcal{L}$ is a localizing subcategory, $\mathcal{L}^{\perp}$ is triangulated and stable for products, i.e. whenever a product of objects of $\mathcal{L}^{\perp}$ exists in $\mathrm{T}$, it belongs to $\mathcal{L}^{\perp}$.

Proof We see that the mapping cone of a map between objects in $\mathcal{L}^{\perp}$ is local considering the long exact sequence associated to $\operatorname{Hom}_{\mathrm{T}}(X,-)$, where $X$ is in $\mathcal{L}$. To show that a product $\prod_{i \in I} Y_{i}$ belongs to $\mathcal{L}^{\perp}$ if every $Y_{i}$ does, it is enough to consider that $\operatorname{Hom}\left(X, \prod Y_{i}\right)=$ $\prod \operatorname{Hom}\left(X, Y_{i}\right)$ and this last object is 0 if $X$ belongs to $\mathcal{L}$.

Lemma 1.4 Given two distinguished triangles and maps in $\mathrm{T}$ :

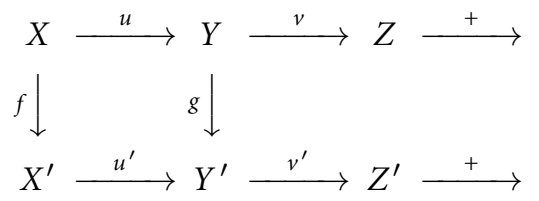

such that $u^{\prime} \circ f=g \circ u$, if $\operatorname{Hom}\left(X, Z^{\prime}[-1]\right)=0$, there is a unique way to complete it to a map of triangles.

Proof Well-known, a written proof is in [BBD, (1.1.9)].

Lemma 1.5 Let $\mathbf{T}$ be a triangulated category, $\mathcal{L}$ a localizing subcategory, and $Y \in \mathcal{L}^{\perp}$. The functor $Q: \mathbf{T} \rightarrow \mathbf{T} / \mathcal{L}$ induces an isomorphism:

$$
\operatorname{Hom}_{\mathrm{T}}(X, Y) \simeq \operatorname{Hom}_{\mathrm{T} / \mathcal{L}}(Q(X), Q(Y))
$$

for every $X \in \mathbf{T}$.

Proof See [Ve, Chap. 1, Section 2, no. 5-3] (or [BN, Lemma 2.9]).

The following proposition gives us a list of useful equivalent characterizations of localizations. They will be used to determine them in different contexts. Also, it shows the consequences of the definition of localization for Verdier quotients by localizing subcategories of $\mathbf{T}$ and the corresponding subcategories of local objects.

Proposition 1.6 Let $\mathrm{T}$ be a triangulated category and $\mathcal{L}$ a localizing subcategory. Denote by $i: \mathcal{L} \rightarrow \mathrm{T}$ and $j: \mathcal{L}^{\perp} \rightarrow \mathrm{T}$ the canonical inclusions and by $Q: \mathrm{T} \rightarrow \mathrm{T} / \mathcal{L}$ the quotient functor. Let ${ }^{\perp}\left(\mathcal{L}^{\perp}\right)=\left\{Z \in \mathbf{T} / \operatorname{Hom}_{\mathbf{T}}(Z, Y)=0\right.$ for every $\left.Y \in \mathcal{L}^{\perp}\right\}$. The following are equivalent: 
(i) There is a localization functor $\ell$ whose associated localizing subcategory is $\mathcal{L}$.

(ii) The functor $Q$ possesses a right adjoint.

(iii) The composition $Q j$ is an equivalence of categories.

(iv) The functor $j$ possesses a left adjoint and $\perp^{\perp}\left(\mathcal{L}^{\perp}\right)=\mathcal{L}$.

(v) The functor $i$ possesses a right adjoint.

(vi) For every $M \in \mathbf{T}$ there is a distinguished triangle

$$
N_{M} \longrightarrow M \longrightarrow B_{M} \stackrel{+}{\longrightarrow}
$$

such that $N_{M} \in \mathcal{L}$ and $B_{M} \in \mathcal{L}^{\perp}$.

Proof (i) $\Rightarrow$ (ii). The functor $\ell$ transforms $\mathcal{L}$-quasi-isomorphisms into isomorphisms. Therefore, by the universal property of $Q, \ell$ factors as $\mathbf{T} \stackrel{Q}{\rightarrow} \mathbf{T} / \mathcal{L} \stackrel{R}{\rightarrow} \mathbf{T}$. The functor $R$ is right adjoint to $Q$. Indeed, $\eta$ : id $\rightarrow \ell=R Q$ is the unit map. On the other hand, if $M^{\prime} \in \mathbf{T} / \mathcal{L}$, there is $M \in \mathbf{T}$ such that $Q M=M^{\prime}$. Applying $Q$ to the map $M \rightarrow \ell M$ we get an isomorphism whose inverse is the desired map $Q R M^{\prime} \rightarrow M^{\prime}$.

(ii) $\Rightarrow$ (iii). Let $R: \mathbf{T} / \mathcal{L} \rightarrow \mathrm{T}$ be the right adjoint to $Q$; we have for $X \in \mathcal{L}$ and $Y \in \mathbf{T}$ that

$$
\operatorname{Hom}_{\mathrm{T}}(X, R Q Y) \simeq \operatorname{Hom}_{\mathrm{T} / \mathcal{L}}(Q X, Q Y)=0
$$

then $R$ factors as $\mathbf{T} / \mathcal{L} \stackrel{r}{\rightarrow} \mathcal{L}^{\perp} \stackrel{j}{\rightarrow} \mathbf{T}$. The functors $Q j$ and $r$ are inverse equivalences. To check this we have a chain of isomorphisms for every $X, Y \in \mathcal{L}^{\perp}$ :

$$
\begin{aligned}
\operatorname{Hom}_{\mathcal{L} \perp}(X, r Q j Y) & =\operatorname{Hom}_{\mathrm{T}}(j X, R Q j Y) \\
& \cong \operatorname{Hom}_{\mathbf{T} / \mathcal{L}}(Q j X, Q j Y) \\
& \cong \operatorname{Hom}_{\mathrm{T}}(j X, j Y) \\
& =\operatorname{Hom}_{\mathcal{L} \perp}(X, Y)
\end{aligned}
$$

where we use Lemma 1.5. For $X^{\prime}$ and $Y^{\prime} \in \mathbf{T} / \mathcal{L}$ such that $Q X=X^{\prime}$ for a certain $X \in \mathbf{T}$, we have:

$$
\begin{aligned}
\operatorname{Hom}_{\mathrm{T} / \mathcal{L}}\left(X^{\prime}, Q j r Y^{\prime}\right) & =\operatorname{Hom}_{\mathrm{T} / \mathcal{L}}\left(Q X, Q R Y^{\prime}\right) \\
& \cong \operatorname{Hom}_{\mathrm{T}}\left(X, R Y^{\prime}\right) \\
& \cong \operatorname{Hom}_{\mathrm{T} / \mathcal{L}}\left(Q X, Y^{\prime}\right) \\
& =\operatorname{Hom}_{\mathrm{T} / \mathcal{L}}\left(X^{\prime}, Y^{\prime}\right)
\end{aligned}
$$

(iii) $\Rightarrow$ (iv). Using again Lemma 1.5, denoting by $r$ the quasi-inverse of $Q j$, we see that $r Q$ is left adjoint to $j$. Furthermore, given $X \in{ }^{\perp}\left(\mathcal{L}^{\perp}\right)$, for any $Y \in \mathbf{T}$

$$
\begin{aligned}
\operatorname{Hom}_{\mathrm{T} / \mathcal{L}}(Q X, Q Y) & \cong \operatorname{Hom}_{\mathcal{L} \perp}(r Q X, r Q Y) \\
& \cong \operatorname{Hom}_{\mathbf{T}}(X, j r Q Y)=0
\end{aligned}
$$


therefore $Q X=0$, i.e., $X \in \mathcal{L}$.

(iv) $\Rightarrow(\mathrm{v})$. Let $j^{\prime}: \mathbf{T} \rightarrow \mathcal{L}^{\perp}$ be the left adjoint to $j$. We complete the unit of the adjunction for $X \in \mathbf{T}$ to a distinguished triangle

$$
X \longrightarrow j j^{\prime} X \longrightarrow N \stackrel{w}{\longrightarrow} X[1]
$$

If $Y \in \mathcal{L}^{\perp}$, we have that

$$
\operatorname{Hom}_{\mathbf{T}}(X, j Y) \cong \operatorname{Hom}_{\mathcal{L} \perp}\left(j^{\prime} X, Y\right) \cong \operatorname{Hom}_{\mathbf{T}}\left(j j^{\prime} X, j Y\right)
$$

Therefore, applying $\operatorname{Hom}_{\mathbf{T}}(-, j Y)$ to the previous triangle we see that $\operatorname{Hom}_{\mathbf{T}}(N, j Y)=0$, so $N \in{ }^{\perp}\left(\mathcal{L}^{\perp}\right)=\mathcal{L}$.

Now, using Lemma 1.4, there is a functor $i^{\prime}: \mathbf{T} \rightarrow \mathcal{L}$ adjoint to $i$ such that $i i^{\prime}(X)=$ $N[-1]$, with counit $\zeta:=-w[-1]$.

$(v) \Rightarrow(v i)$. Again, we have a triangle based on the counit of the adjunction:

$$
i i^{\prime} M \stackrel{\zeta}{\longrightarrow} M \longrightarrow B \longrightarrow i i^{\prime} M[1]
$$

For every $X \in \mathcal{L}, \operatorname{Hom}_{\mathbf{T}}(i X, \zeta)$ is an isomorphism, which implies $\operatorname{Hom}_{\mathbf{T}}(i X, B)=0$, and $B \in \mathcal{L}^{\perp}$.

(vi) $\Rightarrow$ (i). Using again Lemma 1.4 , the assignation $M \mapsto B_{M}$ is functorial. We denote this functor by $\ell$. It comes with a natural transformation $\eta: i d_{\mathrm{T}} \rightarrow \ell$. It follows from (vi) that the objects $X$ such that $\ell X=0$ are precisely those of $\mathcal{L}$. Moreover, an object $Y$ is $\mathcal{L}$ local if, and only if, $\eta_{Y}$ is an isomorphism, so we deduce that $\ell \eta_{Y}=\eta_{\ell Y}$ is an isomorphism from $\ell$ to $\ell^{2}$.

The equivalence between (i) and (vi) was known to Bousfield and Adams and used in [Bo1], the rest of the statements are taken from [Ve, Chap. 1, Section 2, no. 6].

A consequence of the previous result is that if we consider for a Bousfield localization $\ell$ the triangle $N \rightarrow X \rightarrow \ell X \stackrel{+}{\rightarrow}$, then the object $N$ belongs to $\mathcal{L}$ and this assignment defines by the previous result, a functor $\ell^{a}: \mathbf{T} \rightarrow \mathbf{T}$ giving us a functorial distinguished triangle:

$$
\ell^{a} X \longrightarrow X \longrightarrow \ell \stackrel{+}{\longrightarrow}
$$

with $\ell^{a} X \in \mathcal{L}$ and $\ell X \in \mathcal{L}^{\perp}$.

Proposition 1.7 The definition above defines a natural transformation, $\zeta: \ell^{a} \rightarrow 1$ whose formal properties are dual to $\eta$ 's.

Proof Left to the reader. 


\section{Homotopy Limits of Complexes}

From now on, $\mathcal{A}$ will be a Grothendieck category, that means $\mathcal{A}$ is an abelian category with a generator $U$ and exact (set-indexed) filtered direct limits. We will denote by $\mathbf{C}(\mathcal{A})$ the category of complexes of objects of $\mathcal{A}$, which is also a Grothendieck category. As usual $\mathbf{K}(\mathcal{A})$ and $\mathbf{D}(\mathcal{A})$ denote the homotopy and derived categories of $\mathcal{A}$, with their usual structure of triangulated categories.

Our construction of homotopy limits will be based on the totalization of a certain bicomplex naturally associated to a filtered directed diagram. We will fix first the conventions that will be used about bicomplexes and totalization. A bicomplex in an additive category will mean a "complex of complexes", i.e., a family $\left\{B, d_{1}, d_{2}\right\}$ such that $B$ is a bigraded object, $d_{1}^{i j}: B^{i j} \rightarrow B^{i+1 j}$ and $d_{2}^{i j}: B^{i j} \rightarrow B^{i j+1}$ such that $d_{1} \circ d_{1}=0$, $d_{2} \circ d_{2}=0$ and $d_{1} \circ d_{2}=d_{2} \circ d_{1}$, that is, differentials commute. With this convention, the totalization complex is $\operatorname{Tot}(B)^{n}:=\bigoplus_{i+j=n} B^{i j}$, with differential defined by the formula $d^{n}:=\sum_{i+j=n} d_{1}^{i j}+(-1)^{i} d_{2}^{i j}$ (see [I, Section I.11], where morphisms and homotopies of bicomplexes are also defined).

Next, let $G=\left\{G_{s}, \mu_{s t} / s, t \in \Gamma\right\}$ be a filtered directed system in $\mathbf{C}(\mathcal{A})$. It is convenient to introduce the following notation: given $k>0$ and $s \in \Gamma, W_{s}^{k}$ is the set of chains in the ordered set $\Gamma$ of length $k$ which start in $s$. A typical element of $W_{s}^{k}$ will be written $w=\left(s<s_{1}<\cdots<s_{k}\right)$. With this data, we construct a bicomplex $B(G)=\left(B(G), d_{1}, d_{2}\right)$. For $j, k \in \mathbb{Z}$ define:

$$
B(G)^{k j}:= \begin{cases}0 & \text { if } k>0 \\ \bigoplus_{s \in \Gamma} G_{s}^{j} & \text { if } k=0 \\ \bigoplus_{s \in \Gamma, w \in W_{s}^{-k}} G_{s, w}^{j} & \text { if } k<0\end{cases}
$$

where by $G_{s, w}^{j}$ we denote $G_{s}^{j}$ indexed by a (fixed) chain $w$ of $W_{s}^{-k}$. An "element" of $G_{s, w}^{j}$ (i.e., a map $\left.x: U \rightarrow G_{s, w}^{j}\right)$ will be denoted by $(x ; w)$ or $\left(x ; s<s_{1}<\cdots<s_{-k}\right)$. For $k<0$ the horizontal differential $d_{1}$ is defined by the following formula:

$$
\begin{aligned}
d_{1}^{k j}\left(x ; s<s_{1}<\cdots<s_{-k}\right):= & \left(\mu_{s s_{1}}(x) ; s_{1}<\cdots<s_{-k}\right) \\
& +\sum_{i=1}^{-k}\left((-1)^{i} x ; s<\cdots<\check{s}_{i}<\cdots<s_{-k}\right)
\end{aligned}
$$

where the symbol $\check{s_{i}}$ means that $s_{i}$ is suppressed from the chain. If $k \geq 0$ then $d_{1}$ is clearly 0 . We use the standard abuse of pretending we are dealing with actual elements, but the conscientious reader could substitute this with maps from the generator $U$. It is easy but tedious to check that $d_{1} \circ d_{1}=0$. The vertical differential $d_{2}$ is induced by the differential of the complexes $G_{s}$, so it is obvious that $d_{2} \circ d_{2}=0$. Finally, it can be seen that $d_{1} \circ d_{2}=d_{2} \circ d_{1}$ using that the maps $\mu_{\text {st }}$ commute with $d_{2}$.

Definition Let $G=\left\{G_{s} / s \in \Gamma\right\}$ be a filtered direct system of complexes, we define the homotopy direct limit of the system as the totalization of the bicomplex $B(G)$, and we denote it by $\underset{s \in \Gamma}{\operatorname{holim}} G_{s}$. 
Our next task will be to justify the name of this construction, we will see that in fact that it provides another means to compute the homology of the direct limit complex.

Lemma 2.1 Let $F$ be a finite ordered set with maximum $m$. Let $G=\left\{G_{s}, \mu_{s, t} / s, t \in F\right\}$ be a directed system in $\mathbf{C}(\mathcal{A})$. The complex $G_{m}$ is homotopically equivalent to $\underset{s \in F}{\text { holim }} G_{s}$.

Proof The inclusion $G_{m} \rightarrow B(G)^{0}=\bigoplus_{s \in F} G_{s}$ defines a canonical map $\psi: G_{m} \rightarrow$ $\underset{\text { holim }}{\longrightarrow} G_{s}$. Consider the map $\oplus_{s \in F} G_{s} \rightarrow G_{m}$ defined by $\mu_{s m}$ in each component (by conven$\overrightarrow{s \in F}$ tion, $\left.\mu_{s s}=i d\right)$. It gives us a map $\phi$ in the opposite direction. It is clear that $\phi \circ \psi=i d$. On the other hand, let us define a homotopy $h$ of $\underset{s \in F}{\operatorname{holim}} G_{s}$, by the following formula:

$$
\begin{gathered}
h^{j}: \underset{k \leq 0, s \in \Gamma, w \in W_{s}^{-k}}{G_{s, w}^{j-k} \longrightarrow} \bigoplus_{k \leq 0, s \in \Gamma, w \in W_{s}^{-k+1}} G_{s, w}^{j-k} \\
h^{j}\left(x ; s<s_{1}<\cdots<s_{-k}\right)= \begin{cases}0 & \text { if } s_{-k}=m \\
(-1)^{k}\left(x ; s<s_{1}<\cdots<s_{-k}<m\right) & \text { if } s_{-k} \neq m .\end{cases}
\end{gathered}
$$

By looking at each summand $G_{s, w}^{j-k}$ with $w=\left(s<s_{1}<\cdots<s_{-k}\right)$, the reader can check that $\psi \phi-i d=h d+d h$, as required.

Remark The construction of the homotopy $h$ is a generalization of the one that gives the homotopy equivalence between the mapping cylinder of a map $X \rightarrow Y$ and $Y$. In fact, taking as our direct system $G_{0}=X$ and $G_{1}=Y$, our $h$ is the usual homotopy, $c f$. [I, p. 25].

A general (filtered) direct system is a direct limit of its finite subsystems with maximum. One could tend to think that the previous statement generalizes by taking limits. Unfortunately, the maps $h$ are not compatible with this system because they point to the maximum of $F$ which is not "recognizable" inside a big limit diagram. However, they induce quasiisomorphisms that are indeed compatible with direct limits, because in our setting they are exact, so we obtain:

Theorem 2.2 Let $G=\left\{G_{s}, \mu_{s, t} / s, t \in \Gamma\right\}$ be a directed system in $\mathrm{C}(\mathcal{A})$. There is a quasiisomorphism (i.e., an isomorphism in $\mathbf{D}(\mathcal{A})$ )

$$
\phi: \underset{s \in \Gamma}{\operatorname{holim}} G_{s} \rightarrow \underset{s \in \Gamma}{\lim } G_{s}
$$

Proof Denote by $M(\Gamma)$ the set of finite subsets of $\Gamma$ with maximum. It is a filtered set ordered by inclusion. If $F \in M(\Gamma)$, we denote by $G_{F}=\left\{G_{s} / s \in F\right\}$ the direct $F$-system induced by $G$. Then

$$
\underset{F \in M(\Gamma)}{\lim _{F}} \operatorname{Tot}\left(B\left(G_{F}\right)\right)=\operatorname{Tot}(B(G)) .
$$


By the previous proposition there is a map of complexes

$$
\phi_{F}: \operatorname{Tot}\left(B\left(G_{F}\right)\right)=\underset{s \in F}{\operatorname{holim}} G_{s} \rightarrow G_{m_{F}}
$$

where $m_{F}=\max (F)$, that is a quasi-isomorphism. The maps $\phi_{F}$ are compatible with the system $M(\Gamma)$ therefore, taking limits, we obtain that $\operatorname{Tot}(B(G))$ is quasi-isomorphic to $\lim _{s \in \Gamma} G_{s}$.

\section{Homotopy Limits and Localizing Subcategories}

The main result of this section is the following:

Theorem 3.1 Let $\mathcal{L}$ be localizing subcategory of $\mathbf{D}(\mathcal{A})$, let $\Gamma$ be a filtered ordered set and $\left\{G_{s} / s \in \Gamma\right\}$ be a directed system in $\mathbf{C}(\mathcal{A})$ such that $G_{s}$ belongs to $\mathcal{L}$ for everys $\in \Gamma$. Then,

$$
\underset{s \in \Gamma}{\operatorname{holim}} G_{s}
$$

also belongs to $\mathcal{L}$.

We will decompose the proof in a series of intermediate results, maybe of independent interest. We say that a map is semi-split whenever it is split as a map of graded objects. We begin constructing a semi-split filtration of the totalization of a horizontally boundedabove bicomplex.

Lemma 3.2 Let $\left\{B^{*}, d_{1}, d_{2}\right\}$ be a bicomplex in $\mathcal{A}$ such that $B^{i \cdot}=0$ for every $i>i_{0}$. The complex $\operatorname{Tot}\left(B^{*}\right)$ has a semi-split, exhaustive, increasing filtration of complexes $F_{n}^{*}$ such that the successive quotients are $F_{n}^{*} / F_{n-1}^{\cdot}=B^{-n \cdot}[n]$. By exhaustive we mean that:

$$
\lim _{n \in \mathbb{N}} F_{n}^{\cdot}=\operatorname{Tot}\left(B^{*}\right)
$$

the limit taken, obviously, in $\mathbf{C}(\mathcal{A})$.

Proof After shifting, we may assume that $i_{0}=0$. The only special use is made of this integer is to have a place where to begin the induction. Let $F_{0}:=B^{0}$. We will construct the filtration inductively. Suppose $F_{n}$ is already constructed, together with a semi-split exact sequence

$$
F_{n-1}^{\cdot} \stackrel{\mu_{n-1}}{\longrightarrow} F_{n}^{\cdot} \stackrel{w_{n}}{\longrightarrow} B^{-n \cdot[n] .}
$$

It will be clear from the construction that this sequence comes from a standard triangle in $\mathbf{D}(\mathcal{A})$ (i.e., one where the third object is the cone of the first map):

$$
B^{-n \cdot}[n-1] \stackrel{h_{n-1}}{\longrightarrow} F_{n-1}^{\cdot} \stackrel{\mu_{n-1}}{\longrightarrow} F_{n}^{\cdot} \stackrel{w_{n}}{\longrightarrow} B^{-n \cdot}[n] .
$$


Denote by $\nu_{n}$ the usual splitting (as graded objects) of $w_{n}$, and define $h_{n}$ as the following composition:

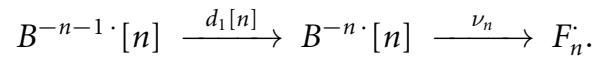

Using that, by construction, $F_{n}$ is the mapping cone of $h_{n-1}$, this gives us an explicit expression for $\nu_{n}$ which allows us to check that $h_{n}$ is an actual map of complexes. Define $F_{n+1}$ as the mapping cone of $h_{n}$. This gives us the desired filtration. Indeed, as graded objects it is clear that $F_{n}^{\cdot}$ is a subobject of $\operatorname{Tot}\left(B^{*}\right)$ for every $n \in \mathbb{N}$. By induction, we see that the canonical inclusion $\eta_{n}: F_{n} \rightarrow \operatorname{Tot}\left(B^{*}\right)$ commutes with differentials, and moreover $\eta_{n}=\eta_{n+1} \circ \mu_{n}$, so the limit makes also sense as complexes. As graded objects, $F_{n}^{j}=\bigoplus_{k=0}^{n} B^{-k j+k}$, therefore,

$$
\lim _{n \in \mathbb{N}} F_{n}^{j}=\bigoplus_{k \in \mathbb{N}} B^{-k j+k}=\operatorname{Tot}\left(B^{*}\right)^{j}
$$

as it was claimed.

Remark This lemma was inspired by [Bo2a]. In this note, Bousfield repairs a mistake in his construction of non-countable homotopy limits that appears in [Bo1] and [Bo2]. In line 10, he says "Thus $C$ is a CW-spectrum with an increasing filtration ... " and this was the clue to the statement of Lemma 3.2. The fact that homotopy limits cannot be constructed from a single triangle makes its generalization difficult to general triangulated categories, because totalizations might not exist and even if they exist, they might not be unique. Of course the situation is easier in the countable case, where the construction can be expressed by a single triangle (see $[\mathrm{BN}]$ ).

3.3 We recall now a construction of countable direct limits due to Milnor. Suppose we have a directed system $\left\{G_{n} / n \in \mathbb{N}\right\}$ in $\mathbf{C}(\mathcal{A})$. To give it, it is enough to know the maps $\mu_{n}: G_{n} \rightarrow G_{n+1}$. Consider the maps $1-\mu_{n}: G_{n} \rightarrow G_{n} \oplus G_{n+1}$. They define a map:

$$
\bigoplus_{n \in \mathbb{N}} G_{n} \longrightarrow \bigoplus_{n \in \mathbb{N}} G_{n}
$$

which we denote symbolically as $1-\mu$. Its cokernel is clearly identified with $\underset{n \in \mathbb{N}}{\lim } G_{n}$.

Lemma 3.4 The map $1-\mu$ is monic.

Proof Left to the reader.

Lemma 3.5 Let $G=\left\{G_{n} / n \in \mathbb{N}\right\}$ be a directed system in $\mathbf{C}(\mathcal{A})$. Let $\mathcal{L}$ be a localizing subcategory of $\mathbf{D}(\mathcal{A})$. If $G_{n} \in \mathcal{L}$, for every $n \in \mathbb{N}$, then $\underset{n \in \mathbb{N}}{\lim _{n}} G_{n} \in \mathcal{L}$. 
Proof Consider the Milnor exact sequence:

$$
0 \rightarrow \bigoplus_{n \in \mathbb{N}} G_{n} \stackrel{1-\mu}{\longrightarrow} \bigoplus_{n \in \mathbb{N}} G_{n} \longrightarrow \underset{n \in \mathbb{N}}{\lim _{\vec{N}}} G_{n} \rightarrow 0 .
$$

This gives us a distinguished triangle in $\mathbf{D}(\mathcal{A})$,

$$
\bigoplus_{n \in \mathbb{N}} G_{n} \stackrel{1-\mu}{\longrightarrow} \bigoplus_{n \in \mathbb{N}} G_{n} \longrightarrow \underset{n \in \mathbb{N}}{\lim _{n}} G_{n} \stackrel{+}{\rightarrow},
$$

whence the conclusion.

Proof of Theorem 3.1 By definition,

$$
\underset{s \in \Gamma}{\operatorname{holim}} G_{s}=\operatorname{Tot}\left(B(G)^{\cdot \cdot}\right) \text {, }
$$

where $B(G)^{\text {' }}$ is a bicomplex whose columns $B(G)^{i}$ are direct sums of the objects in the system, so they belong to $\mathcal{L}$. By Lemma 3.2,

$$
\operatorname{Tot}\left(B(G)^{\cdot}\right)=\underset{n \in \mathbb{N}}{\lim _{n}} F_{n}
$$

where $F_{n}$ is a the cone of a map from a (shift of a) column of $B(G)^{\cdots}$ to $F_{n-1}$, which belongs to $\mathcal{L}$ by induction, therefore $F_{n} \in \mathcal{L}$. We conclude by the previous result.

\section{Localization in the Derived Category of a Ring and K-Projective Resolu- tions}

In this section we prove our main theorem for the case of (unbounded) derived categories of modules. In the path to the proof we will show the existence of unbounded projective resolutions whenever the base category $\mathcal{A}$ has a projective generator.

Let us fix some terminology. We continue to denote by $\mathcal{A}$ a Grothendieck category, $\mathbf{K}(\mathcal{A})$ and $\mathbf{D}(\mathcal{A})$ its homotopic and derived categories, respectively. Let $U$ be a generator of $\mathcal{A}$. Denote by $Z$ the full triangulated subcategory of $\mathbf{K}(\mathcal{A})$ whose objects are acyclic complexes. It is a thick subcategory (i.e., stable for direct summands) whose Verdier quotient is precisely $\mathbf{K}(\mathcal{A}) / Z=\mathbf{D}(\mathcal{A})$. The subcategory $Z$ is clearly localizing because coproducts are exact in $\mathcal{A}$. A $\mathcal{Z}$-quasi-isomorphism is called, as usual, a quasi-isomorphism. We call the local objects for $Z, K$-injective complexes, we refer to [BN] and [Sp] for their elementary properties. Also we call the Z-colocal objects $K$-projectives. By Z-colocal we mean a complex $P$ such that $\operatorname{Hom}_{\mathbf{K}(\mathcal{A})}(P, X)=0$ for every $X$ in $Z$. As a consequence, the full subcategory of K-projective complexes of $\mathbf{K}(\mathcal{A})$ is a localizing subcategory.

We begin with some lemmas.

Lemma 4.1 Let $E$ be an object of a triangulated category $\mathbf{T}$ with coproducts. Let $\mathcal{L}$ be the smallest localizing subcategory that contains $E$. The group $\operatorname{Hom}_{\mathrm{T}}(E[j], B)=0$ for every $j \in \mathbb{Z}$ if, and only if, B is L-local. 
Proof The "if" part is trivial. Let us prove the "only if" part. Suppose that $B \in \mathbf{T}$ is such that $\operatorname{Hom}_{\mathrm{T}}(E[j], B)=0$ for every $j \in \mathbb{Z}$, we have to show that $\operatorname{Hom}_{\mathrm{T}}(X, B)=0$ for every $X \in \mathcal{L}$. Define $S$ as the full subcategory of $\mathcal{L}$ whose objects $X$ are such that $\operatorname{Hom}_{\mathrm{T}}(X[j], B)=0$, for every $j \in \mathbb{Z}$. $\mathbf{S}$ is clearly stable for shiftings and if two objects of a distinguished triangle are in $\mathbf{S}$, so does the third, by the long exact sequence. Finally, if $\left\{X_{i} / i \in I\right\}$ is a family of objects of $\mathbf{S}, \bigoplus_{i \in I} X_{i}$ is in $\mathbf{S}$, because

$$
\operatorname{Hom}_{\mathrm{T}}\left(\bigoplus_{i \in I} X_{i}[j], B\right) \simeq \prod_{i \in I} \operatorname{Hom}_{\mathrm{T}}\left(X_{i}[j], B\right)=0 .
$$

By hypothesis, $E$ belongs to $S$, therefore $S=\mathcal{L}$.

Lemma 4.2 With the previous notations, let $\mathcal{L}$ the smallest localizing subcategory of $\mathbf{K}(\mathcal{A})$ containing $U$, then $\mathcal{L}^{\perp} \subset Z$. If moreover $U$ is projective (in $\mathcal{A}$ ), then $\mathcal{L}^{\perp}=Z$.

Proof Given a complex $(F, d)$ of $\mathcal{A}$, we have a canonical isomorphism

$$
\operatorname{Hom}_{\mathbf{C}(\mathcal{A})}(U[-i], F) \stackrel{\sim}{\longrightarrow} \operatorname{Hom}_{\mathcal{A}}\left(U, \operatorname{ker}\left(d^{i}\right)\right) \quad(i \in \mathbb{Z})
$$

which carries null-homotopic morphisms to maps $U \rightarrow \operatorname{ker}\left(d^{i}\right)$ that factor through $F^{i-1}$. Therefore, if $F \in \mathcal{L}^{\perp}$ all these maps are 0 , so $F$ has to be exact because $U$ is a generator.

Conversely, suppose further $F$ acyclic and $U$ a projective object, then the map

$$
\operatorname{Hom}_{\mathcal{A}}\left(U, F^{i-1}\right) \longrightarrow \operatorname{Hom}_{\mathcal{A}}\left(U, \operatorname{ker}\left(d^{i}\right)\right)
$$

is onto for every $i \in \mathbb{Z}$, which implies $\operatorname{Hom}_{\mathbf{K}(\mathcal{A})}(U[-i], F)=0$.

Proposition 4.3 If $U$ is a projective object of $\mathcal{A}$, then every complex has a $K$-projective resolution. In other words, there is a localization functor such that the associated localizing subcategory is the full subcategory of K-projective complexes.

Proof Let $\mathcal{L}$ the smallest localizing subcategory of $\mathbf{K}(\mathcal{A})$ containing $U$. A bounded above complex all of whose objects are direct sums of $U$ is easily seen to be K-projective, therefore by the well-known step-by-step process to every bounded above complex, we are able to construct a K-projective resolution. Let $M$ be any object of $K(\mathcal{A})$. Let $M \leq n$ denote the truncation of $M$ on the level $n$ from above. For every $n \in \mathbb{N}$, there is quasi-isomorphism $g_{n}: P_{n} \stackrel{\sim}{\rightarrow} M^{\leq n}$, where $P_{n}$ is K-projective. The canonical map $M^{\leq n} \rightarrow M^{\leq n+1}$ induces a map $P_{n} \rightarrow P_{n+1}$, so $\left\{P_{n}\right\}_{n \in \mathbb{N}}$ is a countable direct system of complexes. Consider an induced map of triangles in $\mathbf{K}(\mathcal{A})$ :

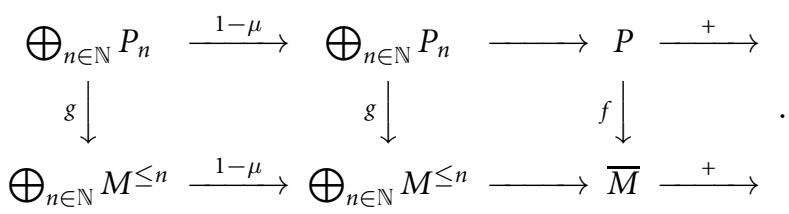


Every $P_{n}$ is in $\mathcal{L}$, therefore $\bigoplus_{n \in \mathbb{N}} P_{n} \in \mathcal{L}$ and $P \in \mathcal{L}$. It is clear that $f$ is a quasiisomorphism because so is $g$. Furthermore, the canonical map of complexes $h: \bar{M} \rightarrow$ $\lim _{n \in \mathbb{N}} M \leq n=M$ is a quasi-isomorphism. So $M$ sits in a triangle:

$$
P \stackrel{h \circ f}{\longrightarrow} M \longrightarrow B \longrightarrow P[1]
$$

where $P$ is $\mathrm{K}$-projective and $B$ is an acyclic complex.

Remark If $\mathcal{A}=R$-mod, then the $\operatorname{ring} R$ is a projective small generator of $\mathcal{A}$, so the previous result is a slight generalization of the well-known theorem on the existence of $\mathrm{K}$-projective resolutions of complexes of $R$-modules. Our proof follows the standard one as in $[\mathrm{BN}]$. Observe that K-projective resolutions are the acyclization functor associated to the localization with respect to $Z$. It has also been remarked by Keller and Weibel that old-fashioned Cartan-Eilenberg resolutions also work due to the exactness of coproducts for modules.

Now we will prove Bousfield localization for derived categories of modules, as a first step towards the general Theorem 5.7 for arbitrary Grothendieck categories. Let $R$ be a ring. Denote by $R$-mod the category of left $R$-modules. As usual, we abbreviate $\mathbf{C}(R$-mod), $\mathbf{K}(R$-mod $)$ and $\mathbf{D}(R$-mod $)$, by $\mathbf{C}(R), \mathbf{K}(R)$ and $\mathbf{D}(R)$. We denote by $Q_{R}: \mathbf{K}(R) \rightarrow \mathbf{D}(R)$ the canonical functor.

4.4 We recall that an ordinal can be identified with a well-ordered set and that a cardinal is a minimum of equipotent ordinals. An ordinal is regular if, as an ordered set, every proper cofinal well-ordered subset is a strictly smaller ordinal. A cardinal is regular if its associated ordinal is. A key fact we will use is Hausdorff's theorem: A successor cardinal is regular. See [L, 3.11 Proposition, p. 135]. This is the set-theoretic key point in the proof of the next result, and also in [Bo1], [Bo2] and the proof of the existence of K-injective resolutions for sheaves in $[\mathrm{Sp}]$. In these references it is used without mention. It should be emphasized that Hausdorff's theorem follows simply from the axiom of choice and does not depend on "exotic" big cardinal axioms.

Proposition 4.5 Let $E \in \mathbf{C}(R)$ and $\mathcal{L}$ the smallest localizing subcategory of $\mathbf{D}(R)$ containing $E$. Then, there exists a localization functor $\ell$ in $\mathbf{D}(R)$ such that $\ell(X)=0 \Longleftrightarrow X \in \mathcal{L}$.

Proof First, we can assume that $E$ is K-projective by Proposition 4.3. Therefore, for $k \in \mathbb{Z}$ every $E[k]$ will also be K-projective and $\operatorname{Hom}_{\mathbf{D}(R)}(E[k], X) \simeq \operatorname{Hom}_{\mathbf{K}(R)}(E[k], X)$ for $X \in \mathbf{C}(R)$, i.e., maps from $E[k]$ in the derived category are represented by actual maps of complexes.

Now, by Proposition 1.6, it is enough, for any $M \in \mathbf{D}(R)$, to construct a distinguished triangle

$$
N_{M} \longrightarrow M \longrightarrow B_{M} \stackrel{+}{\longrightarrow}
$$

such that $N_{M} \in \mathcal{L}$ and $B_{M}$ is $\mathcal{L}$-local. Let $I$ be a well-ordered set. We will construct, by transfinite induction, successive "approximations" of $B_{M}$. More precisely, for every $s \in I$ 
we will construct a complex $B_{s}$ and for $s \leq t \in I$ a semi-split monomorphism of complexes $\mu_{s t}: B_{s} \rightarrow B_{t}$ such that:

(i) $\left\{B_{s}, \mu_{s t} / s, t \in I\right\}$ is a directed system in $\mathbf{C}(R)$.

(ii) If $f \in \operatorname{Hom}_{\mathrm{C}(R)}\left(E[k], B_{s}\right)$ and $s<t$, then $\mu_{s t} \circ f$ is homotopic to zero.

Let 0 be the minimum of $I$, we define $B_{0}:=M$.

If $s \in I$ has a predecessor $s-1$, suppose by induction that $B_{s-1}$ is already constructed. Take $\Omega_{s-1}:=\bigcup_{k \in \mathbb{Z}} \operatorname{Hom}_{\mathbf{C}(R)}\left(E[k], B_{s-1}\right)$, and let $\alpha_{s-1}: \bigoplus_{\Omega_{s-1}} E[k] \rightarrow B_{s-1}$ be the map of complexes given by the universal property of the coproduct. We define $\mu_{s-1 s}: B_{s-1} \rightarrow B_{s}$ as the semi-split monomorphism given by the canonical distinguished triangle:

$$
\bigoplus_{\Omega_{s-1}} E[k] \stackrel{\alpha_{s-1}}{\longrightarrow} B_{s-1} \stackrel{\mu_{s-1 s}}{\longrightarrow} B_{s} \longrightarrow\left(\bigoplus_{\Omega_{s-1}} E[k]\right)[1],
$$

where $B_{s}$ is the cone of $\alpha_{s-1}$. For any $i<s$, let $\mu_{i s}:=\mu_{s-1 s} \circ \mu_{i s-1}$, which is again a semisplit monomorphism. If $f \in \operatorname{Hom}_{\mathbf{C}(R)}\left(E[k], B_{i}\right)$, and $i<s$, then $\mu_{i s} \circ f$ is homotopic to zero by construction if $i=s-1$, and by the inductive hypothesis if $i<s-1$.

If $s \in I$ has no predecessor, take

$$
B_{s}:=\lim _{\overrightarrow{i<s}} B_{i} \text { and for } i<s, \mu_{i s}=\lim _{i<j<s} \mu_{i j} .
$$

Both limits taken, of course, as objects and maps in $\mathbf{C}(R)$. Again, it is clear that it satisfies the needed conditions.

Now we fix $I$ as a set of ordinals. Let $\gamma$ be the least ordinal such that $\#(\gamma)>$ $\# \bigcup_{p \in \mathbb{Z}} \operatorname{Hom}_{\mathbf{C}(R)}(R[p], E)$ and let $I$ be the set of ordinals strictly smaller than $\gamma$. We claim that it is enough to take $B_{M}=B_{\gamma}$. Indeed, let us check first that $B_{M}$ is $\mathcal{L}$-local. By Lemma 4.1 it is enough to see that $\operatorname{Hom}_{\mathbf{D}(R)}\left(E[k], B_{M}\right)=\operatorname{Hom}_{\mathbf{K}(R)}\left(E[k], B_{M}\right)=0$, for each $k \in \mathbb{Z}$. Let $g: E[k] \rightarrow B_{M}$ be a map of complexes, let us show that $g$ is homotopic to zero. Define $B_{i}^{\prime}$ by the following cartesian square

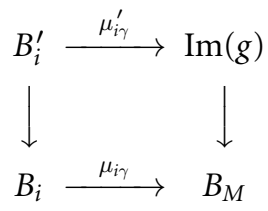

and $\mu_{i s}^{\prime}: B_{i}^{\prime} \rightarrow B_{s}^{\prime}$ with $i<s$, by the universal property of the cartesian square. We have defined a new directed system $\left\{B_{s}^{\prime}, \mu_{s t}^{\prime} / s, t \in I\right\}$ such that:

$$
\underset{\overrightarrow{i<s}}{\lim _{i}} B_{i}^{\prime}=\underset{\overrightarrow{i<s}}{\lim _{\longrightarrow}}\left(B_{i} \cap \operatorname{Im}(g)\right)=B_{M} \cap \operatorname{Im}(g)=\operatorname{Im}(g)
$$

where in the middle identification we have used that $\mathbf{C}(R)$ is a Grothendieck category. We claim that there is an index $s_{0}<\gamma$ such that

$$
\underset{i<s}{\lim _{i<s}} B_{i}^{\prime}=B_{s_{0}}^{\prime}
$$


To see this by reductio ad absurdum, suppose that for every $s \in I$, there is a $t>s$ such that $\mu_{s t}^{\prime}$ is not an epimorphism. Define sets $J_{s}:=\left\{r \geq s / \mu_{r r+1}^{\prime}\right.$ is not an epimorphism $\}$. Each $J_{s}$ is not empty, so $J:=J_{0}$ is a cofinal subset of $I$, but then $\#(J)=\#(\gamma)$, because $\gamma$ is a regular ordinal. On the other hand, we can define a map

$$
\phi: J \rightarrow \bigcup_{p \in \mathbb{Z}} \operatorname{Hom}_{\mathbf{C}(R)}(R[p], \operatorname{Im}(g))
$$

by $\phi(s)=\mu_{s+1 \gamma}^{\prime} \circ h_{s}$ where $h_{s}$ is a map from $R[p]$ to $B_{s+1}^{\prime}$ (an element) that does not factor through $B_{s}^{\prime}$. The map $\phi$ is injective, therefore,

$$
\#(J) \leq \#\left(\bigcup_{p \in \mathbb{Z}} \operatorname{Hom}_{\mathbf{C}(R)}(R[p], \operatorname{Im}(g))\right)<\#(\gamma) .
$$

The last inequality holds because $\operatorname{Im}(g)$ is a quotient of $E[k]$. The fact that $\#(J)<\#(\gamma)$ is a contradiction by Hausdorff's theorem (see 4.4). Thus, there is a $s_{0} \in I$ such that $\mu_{s_{0} \gamma}^{\prime}$ is an isomorphism and then $g$ factors through $\mu_{s_{0} \gamma}$, therefore it is homotopic to zero.

Finally, let us study the third object in the triangle defined by the map $M \rightarrow B_{M}$. For every $i<\gamma$, insert $\mu_{0 i}$ in the distinguished triangle

$$
N_{i} \longrightarrow M \stackrel{\mu_{0 i}}{\longrightarrow} B_{i} \longrightarrow N_{i}[1] .
$$

We can take $N_{i}[1]=\operatorname{Coker}\left(\mu_{0 i}\right)$ (taken in $\left.\mathbf{C}(R)\right)$. So, in the distinguished triangle

$$
N_{M} \longrightarrow M \stackrel{\mu_{0 \gamma}}{\longrightarrow} B_{M} \longrightarrow N_{M}[1]
$$

we have $N_{M}=\underset{\substack{\vec{i} \gamma}}{\lim _{i}} N_{i}$, where for $s<t$ the transition maps $N_{s} \rightarrow N_{t}$ of this system are induced by $\mu_{s t}: B_{s} \rightarrow B_{t}$. To finish the proof we have to prove that $N_{M} \in \mathcal{L}$. By Theorem 2.2 and Theorem 3.1 it is enough to see that $N_{i} \in \mathcal{L}$ for every $i \in I$.

First, $N_{0}=0$, so it is trivially in $\mathcal{L}$. If $i$ has a predecessor, consider the triangles like (1) whose second maps are $\mu_{0 i-1}$ and $\mu_{0 i}$. Consider also the distinguished triangle:

$$
\bigoplus_{\Omega_{i-1}} E[k] \longrightarrow B_{i-1} \stackrel{\mu_{i-1 i}}{\longrightarrow} B_{i} \longrightarrow\left(\bigoplus_{\Omega_{i-1}} E[k]\right)[1] .
$$

We have $\mu_{i-1 i} \circ \mu_{0 i-1}=\mu_{0 i}$, then the octahedral axiom gives us a distinguished triangle

$$
N_{i-1} \longrightarrow N_{i} \longrightarrow \bigoplus_{\Omega_{i-1}} E[k] \longrightarrow N_{i-1}[1]
$$

where $N_{i-1}$ and $\bigoplus_{\Omega_{i-1}} E[k]$ belong to $\mathcal{L}$, the first by induction hypothesis and the second by its form. This implies $N_{i}$ is also in $\mathcal{L}$. Finally, if $i$ has no predecessor, $N_{i}$ is a limit of complexes $N_{s}$ that belong to $\mathcal{L}$ by induction. Applying Theorem 2.2 we have that

$$
\underset{s<i}{\operatorname{holim}} N_{s} \simeq \underset{s<i}{\lim } N_{s}
$$

and by Theorem 3.1, the homotopy limit belongs to $\mathcal{L}$, so we reach the desired conclusion. 


\section{Localization in $\mathbf{D}(\mathcal{A})$ and K-Injective Resolutions}

In this section we will extend Gabriel-Popescu embedding to derived categories. We will obtain as a consequence the existence of K-injective resolutions for complexes in a Grothendieck category and the existence of localizations in its derived category.

By Gabriel-Popescu embedding ([GP], see also [St, Chap. X, Theorem 4.1], there is a ring $R$ (namely, $\operatorname{End}_{\mathcal{A}}(U)$ where $U$ is a generator of $\mathcal{A}$ ), such that $\mathcal{A}$ is a quotient (in the sense of Abelian categories) of $R$-mod by the thick subcategory of torsion objects of a hereditary torsion theory. This means there is a couple of functors

$$
R-\bmod \underset{i}{\stackrel{a}{\rightleftarrows}} \mathcal{A}
$$

where $a$ is an exact functor and $i$ is left-exact full, faithful and right adjoint to $a$. The objects that are sent to zero by $a$ are called torsion objects. This situation extends immediately to homotopy categories

$$
\mathbf{K}(R) \underset{i}{\stackrel{a}{\rightleftarrows}} \mathbf{K}(\mathcal{A})
$$

Because of this, $\mathbf{K}(\mathcal{A})$ is identified with a calculus of fractions of $\mathbf{K}(R)$.

We need to transport (2) to the derived category situation. The extension of the functor $a$ is clear, by exactness. Let us denote this extension by a: $\mathbf{D}(R) \rightarrow \mathbf{D}(\mathcal{A})$. Let $\mathcal{L}_{\mathcal{A}}$ the full subcategory of $\mathbf{D}(R)$ whose objects are $\{X \in \mathbf{D}(R) / \mathrm{a} X=0\}$. The extension of $i$ will be constructed using the previous results. (See 1.6, (i) $\Leftrightarrow$ (ii).)

Proposition 5.1 There is a subset of the class of objects of $\mathbf{D}(R)$ such that the smallest localizing subcategory of $\mathbf{D}(R)$ that contains them is $\mathcal{L}_{\mathcal{A}}$. This identifies $\mathbf{D}(\mathcal{A})$ with the subcategory of $\mathcal{L}_{\mathcal{A}}$-local objects and the associated localization functor factors through a.

Proof The subcategory $\mathcal{L}_{\mathcal{A}}$ is localizing and $\mathbf{D}(\mathcal{A})$ is identified with the quotient category $\mathbf{D}(R) / \mathcal{L}_{\mathcal{A}}$. Indeed, let us show that $\mathbf{D}(\mathcal{A})$ satisfies the "universal" property that characterizes $\mathbf{D}(R) / \mathcal{L}_{\mathcal{A}}$. Let $b: \mathbf{D}(R) \rightarrow \mathbf{T}$ be a functor that sends to zero the objects in $\mathcal{L}_{\mathcal{A}}$. The composed functor $\mathbf{K}(R) \stackrel{Q_{R}}{\longrightarrow} \mathbf{D}(R) \stackrel{b}{\rightarrow} \mathbf{T}$ factors through $a: \mathbf{K}(R) \rightarrow \mathbf{K}(\mathcal{A})$ giving a functor $\bar{b}: \mathbf{K}(\mathcal{A}) \rightarrow \mathbf{T}$. Let $B$ be an acyclic complex in $\mathbf{K}(\mathcal{A})$. Then we see that $\bar{b}(B)=\bar{b}(a i B)=b Q_{R} i(B)=0$. The last identification follows from $Q_{R} i(B) \in \mathcal{L}_{\mathcal{A}}$, because its homologies are torsion.

Let us show now that the localizing subcategory $\mathcal{L}_{\mathcal{A}}$ of $\mathbf{D}(R)$ is generated by a set. Let $\beta:=\max \left\{\#(R), \aleph_{0}\right\}$. A complex of $R$-modules $\left(E, d^{*}\right)$ belongs to the set $L$ if it satisfies the following properties:

(i) $E^{j}=0$, if $j>0$.

(ii) $E^{0}=R$.

(iii) $E^{j}=\bigoplus_{I_{j}} R$, where $\#\left(I_{j}\right) \leq \beta$, if $j<0$.

(iv) $a H^{j}(X)=0, \forall j \in \mathbb{Z}$, i.e., its homologies are torsion. 
Let $\mathcal{L}_{0}$ the smallest localizing subcategory of $\mathbf{D}(R)$ that contains $L$. Let us show that $\mathcal{L}_{\mathcal{A}}=$ $\mathcal{L}_{0}$. It is clear that $\mathcal{L}_{0} \subset \mathcal{L}_{\mathcal{A}}$. Let us see the opposite inclusion. By Proposition 4.5 given $M \in \mathcal{L}_{\mathcal{A}}$ there is a distinguished triangle in $\mathbf{D}(R)$ :

$$
M_{0} \longrightarrow M \longrightarrow M^{\prime} \longrightarrow M_{0}[1]
$$

where $M_{0} \in \mathcal{L}_{0}$ and $M^{\prime} \in \mathcal{L}_{0}^{\perp}$. Observe that $M$ and $M_{0} \in \mathcal{L}_{\mathcal{A}}$, therefore $M^{\prime} \in \mathcal{L}_{\mathcal{A}}$. It is enough to see that $M^{\prime}$ is acyclic which implies $M \simeq M_{0} \in \mathcal{L}_{0}$. Suppose then that $M^{\prime}$ is not acyclic, after shifting we will take $H^{0}\left(M^{\prime}\right) \neq 0$. But $M^{\prime} \in \mathcal{L}_{\mathcal{A}}$, therefore there exists an ideal $\mathfrak{a}$ in $R$, with $a(R / \mathfrak{a})=0$ and a non-zero $R$-linear map $g: R / \mathfrak{a} \rightarrow H^{0}\left(M^{\prime}\right)$, because the subcategory of $R$-mod whose objects go to 0 by $a$ is generated by the quotients $R / \mathfrak{a}$ (cf. [St, Chap. VI, Proposition 3.6]. Using $g$, we will construct a non zero map in $\mathbf{D}(R)$ from a complex in $L$ to $M^{\prime}$. But this is impossible, so $M^{\prime}$ has to be acyclic. Indeed, being $R$ projective the map $g$ extends as:

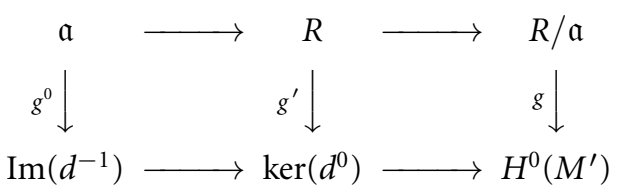

denoting by $d$ the differential of $M^{\prime}$. Let $P^{0}:=R$ and $f^{0}$ be the composition: $R \stackrel{g^{\prime}}{\rightarrow}$ $\operatorname{ker}\left(d^{0}\right) \hookrightarrow M^{\prime 0}$. We will construct a complex $\left(P^{\prime}, d_{P}\right)$ in $L$ with a map $f: P \rightarrow M^{\prime}$ inductively. Suppose everything given up to level $j \leq 0$ such that for $k$ with $j<k<0$, every $P^{k}=\bigoplus_{I_{k}} R$, where $\#\left(I_{k}\right) \leq \beta$. Construct the diagram

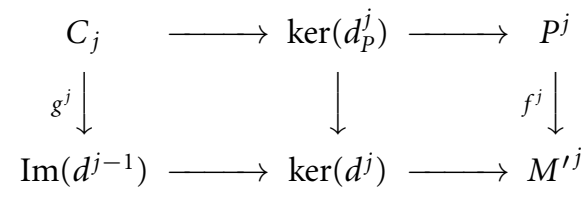

for $j<0$ in such a way that $C_{j}$ makes the left square cartesian. For $j=0$ take (3). We have that $a\left(\operatorname{ker}\left(d_{P}^{j}\right) / C_{j}\right)=0$ because $\operatorname{ker}\left(d_{P}^{j}\right) / C_{j}$ is a submodule of $H^{j}\left(M^{\prime}\right)$ which is torsion by assumption. Observe that $\#\left(C_{j}\right) \leq \#\left(P^{j}\right) \leq \beta$, therefore there is a set $I_{j-1}$ such that $\#\left(I_{j-1}\right) \leq \#\left(C_{j}\right)$ and an epimorphism $p_{j-1}: \bigoplus_{I_{j-1}} R \rightarrow C_{j}$. The module $P^{j-1}:=\bigoplus_{I_{j-1}} R$ is projective so we have a map $f^{j-1}$ fitting in a commutative diagram

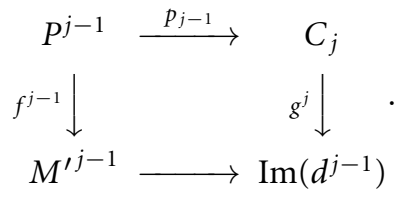

Let $d_{P}^{j-1}$ be the composition $P^{j-1} \rightarrow C_{j} \rightarrow P^{j}$. Now it is clear that $P$ belongs to $L$. Also, $f$ is a map of complexes such that $H^{0}(f)=g \neq 0$ contradicting $\operatorname{Hom}_{\mathbf{D}(R)}\left(P, M^{\prime}\right)=0$.

Corollary 5.2 The functor a: $\mathbf{D}(R) \rightarrow \mathbf{D}(\mathcal{A})$ possesses a right adjoint, which we denote by $\mathrm{i}$. 
Remark There is another approach to the proof of this fact due to Neeman. It uses the Brown representability theorem [N3, Theorem 4.1]. Note that $\mathbf{D}(R)$ is compactly generated (by $R$ ), and the functor a preserves coproducts, so it has a right adjoint. However, you need to know in advance that $\mathbf{D}(\mathcal{A})$ has "small hom-sets" which it is not obvious a priori. In our line of argument, we obtain this fact later as a consequence (see Corollary 5.6).

Proposition 5.3 The canonical functor $Q_{R}: \mathbf{K}(R) \rightarrow \mathbf{D}(R)$ has a right adjoint. In other words: every complex of modules over a ring has a $K$-injective resolution.

Proof The proof is a dualization of that of Proposition 4.3 so we only sketch it. Every bounded below complex has a resolution by a bounded below complex of injective $R$ modules, which is clearly K-injective. In general, given $X \in \mathbf{C}(R)$, for every $n \in \mathbb{N}$, denote by $X^{\geq n}$ the truncation of $X$ on the level $n$ from below and by $g_{n}: X^{\geq-n} \stackrel{\sim}{\rightarrow} I_{n}$ a K-injective resolution. We get a countable inverse system of K-injective complexes $\left\{I_{n}\right\}_{n \in \mathbb{N}}$. We have a diagram of distinguished triangles

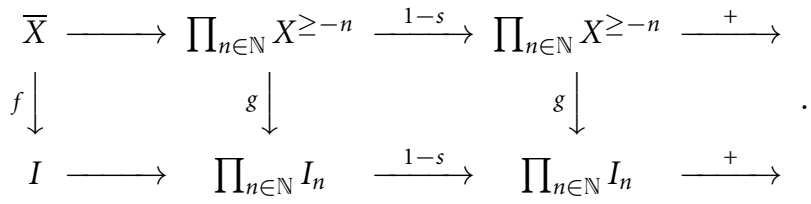

The map $g=\prod_{n \in \mathbb{N}} g_{n}$ is a product of quasi-isomorphisms, therefore a quasi-isomorphism because products are exact in $R$-mod. So the map $f$ is a quasi-isomorphism. Observe that

$$
X=\lim _{n \in \mathbb{N}} X^{\geq-n}
$$

And so there is a morphism $X \rightarrow \prod_{n \in \mathbb{N}} X^{\geq-n}$ that composed with $1-s$ is 0 . Therefore, there is an induced map $h: X \rightarrow \bar{X}$ which is a quasi-isomorphism because the system is Mittag-Leffler. This gives us a triangle in $\mathbf{K}(R)$

$$
N \longrightarrow X \stackrel{f \circ h}{\longrightarrow} I \longrightarrow P[1]
$$

where $I$ is $\mathrm{K}$-injective and $N$ is an acyclic complex.

Remark The existence of K-injective resolutions of complexes of modules over a ring is well known (see [BN, Proposition 2.12]). We have reproduced the proof of Propositions 4.3 and 5.3 here for the convenience of the reader.

The previous discussion can be summarized in a diagram of functors:

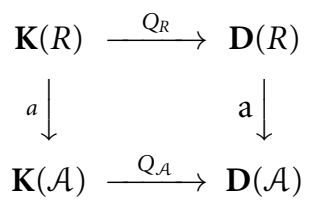

where $Q_{R}, a$ and a have right adjoints, by Proposition 5.3, trivially and Corollary 5.2, respectively. 
Theorem 5.4 The canonical functor $Q_{\mathcal{A}}: \mathbf{K}(\mathcal{A}) \rightarrow \mathbf{D}(\mathcal{A})$ has a right adjoint. In other words: every complex of objects of a Grothendieck category has a K-injective resolution.

Proof Consider the functors

$$
\mathbf{K}(R) \stackrel{a}{\longrightarrow} \mathbf{K}(\mathcal{A}) \stackrel{Q_{\mathcal{A}}}{\longrightarrow} \mathbf{D}(\mathcal{A}) .
$$

Both $a$ and $Q_{\mathcal{A}} \circ a=\mathrm{a} \circ Q_{R}$ have right adjoints by the previous discussion. Moreover, the functor $a$ identifies $\mathbf{K}(\mathcal{A})$ as a calculus of fractions of $\mathbf{K}(R)$ by the discussion preceding Proposition 5.1. So we are in position to apply the next lemma.

Lemma 5.5 Let $\mathbf{T}, \mathbf{K}$ and $\mathbf{D}$ be categories with functors $Q: \mathbf{T} \rightarrow \mathbf{K}$ and $Q^{\prime}: \mathbf{K} \rightarrow \mathbf{D}$. Suppose that $Q$ identifies the category $\mathbf{K}$ as a calculus of fractions of $\mathbf{T}$. If $G=Q^{\prime} \circ Q$ has a right adjoint $k: \mathbf{D} \rightarrow \mathbf{T}$, then so does $Q^{\prime}$, and it is $I:=Q \circ k$.

Proof First of all, note that if $u: Z_{1} \rightarrow Z_{2}$ is a map in $\mathbf{T}$ such that $Q(u)$ is an isomorphism in $\mathbf{K}$, then for $Y \in \mathbf{D}$

$$
\operatorname{Hom}_{\mathbf{T}}\left(Z_{2}, k Y\right) \stackrel{u^{*}}{\longrightarrow} \operatorname{Hom}_{\mathbf{T}}\left(Z_{1}, k Y\right)
$$

is an isomorphism, by the commutativity of the diagram:

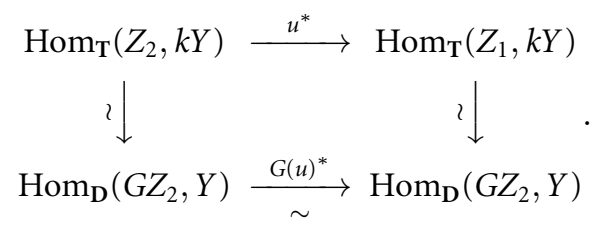

Therefore $Q$ induces natural isomorphisms

$$
\operatorname{Hom}_{\mathbf{T}}(Z, k Y) \stackrel{u^{*}}{\longrightarrow} \operatorname{Hom}_{\mathbf{K}}(Q Z, Q k Y),
$$

for every $Z \in \mathbf{T}, Y \in \mathbf{D}$. Let now $X \in \mathbf{K}$ and $X_{0} \in \mathbf{T}$ such that $Q X_{0}=X$. For any $Y \in \mathbf{D}$ the natural composed isomorphism

$$
\begin{aligned}
\operatorname{Hom}_{\mathbf{D}}\left(Q^{\prime} X, Y\right) & =\operatorname{Hom}_{\mathbf{D}}\left(Q^{\prime} Q X_{0}, Y\right) \\
& =\operatorname{Hom}_{\mathbf{D}}\left(G X_{0}, Y\right) \\
& \cong \operatorname{Hom}_{\mathbf{T}}\left(X_{0}, k Y\right) \\
& \cong \operatorname{Hom}_{\mathbf{K}}\left(Q X_{0}, Q k Y\right) \\
& =\operatorname{Hom}_{\mathbf{K}}(X, I Y)
\end{aligned}
$$

gives us the desired adjunction isomorphism. 
Remark The existence of $\mathrm{K}$-injective resolutions implies that a functor from $\mathbf{K}(\mathcal{A})$ has a right derived functor. Observe that the functor i of Corollary 5.2 is the right derived functor of $i: \mathbf{K}(\mathcal{A}) \rightarrow \mathbf{K}(R)$.

This result shows that there is a localization functor in $\mathbf{K}(\mathcal{A})$ whose associated localizing subcategory are the acyclic complexes, $z$. The result would not follow from a generalization of Theorem 5.7 for homotopy categories because already for $\mathbf{K}(R)$, $Z$ is not generated by a set as is explained in the forthcoming book by Neeman [N4].

Corollary 5.6 If $\mathcal{A}$ is a Grothendieck category, its derived category, $\mathbf{D}(\mathcal{A})$, has "small homsets".

Proof Let $X, Y \in \mathbf{K}(\mathcal{A})$, denote by $Q: \mathbf{K}(\mathcal{A}) \rightarrow \mathbf{D}(\mathcal{A})$ the canonical functor and by $I$ its right adjoint. Then:

$$
\operatorname{Hom}_{\mathbf{D}(\mathcal{A})}(Q X, Q Y) \cong \operatorname{Hom}_{\mathbf{K}(\mathcal{A})}(X, I Q Y)
$$

and this last "hom" is clearly a set.

Remark The general construction does not guarantee that homs in a derived category are sets rather than classes. This problem is addressed for derived categories of modules, presheaves and sheaves in [W, Proposition 10.4.4]. It can be shown directly, but it is an easy consequence of the previous theorem. The question is sometimes referred to as "the existence of the derived category".

Theorem 5.7 Let E be a complex in $\mathbf{D}(\mathcal{A})$. Let $\mathcal{L}$ be the smallest localizing subcategory of $\mathbf{D}(\mathcal{A})$ that contains $E$. There exists a localization functor $\ell$ such that $\ell(X)=0 \Longleftrightarrow X \in \mathcal{L}$.

Proof By Proposition 1.6 it is enough to construct for every $M \in \mathbf{D}(\mathcal{A})$ a distinguished triangle

$$
N \longrightarrow M \longrightarrow B \stackrel{+}{\longrightarrow}
$$

such that $N \in \mathcal{L}$ and $B \in \mathcal{L}^{\perp}$. By Proposition 5.1, there is a localizing subcategory of $\mathbf{D}(R)$ that we denote by $\mathcal{L}_{\mathcal{A}}$ generated by a set of complexes, which after taking the coproduct of all of its objects we can suppose is formed by a single object $F$, such that $\mathbf{D}(R) / \mathcal{L}_{\mathcal{A}}$ is identified with $\mathbf{D}(\mathcal{A})$. Take the notation as in Corollary 5.2 and let $\mathcal{L}^{\prime}$ be the smallest localizing subcategory of $\mathbf{D}(R)$ containing $\{\mathrm{i}(E), F\}$. Then, by Proposition 4.5 , for each $M \in \mathbf{D}(\mathcal{A})$, there is a distinguished triangle in $\mathbf{D}(R)$

$$
N_{0} \longrightarrow \mathrm{i}(M) \longrightarrow B_{0} \stackrel{+}{\longrightarrow}
$$

such that $N_{0} \in \mathcal{L}^{\prime}$ and $B_{0} \in \mathcal{L}^{\prime \perp}$. Applying the functor a, we get a distinguished triangle in $\mathbf{D}(\mathcal{A})$

$$
\mathrm{a} N_{0} \longrightarrow M \longrightarrow \mathrm{a} B_{0} \stackrel{+}{\longrightarrow}
$$


where $\mathrm{a} N_{0} \in \mathcal{L}$. Let us check that $\mathrm{a} B_{0} \in \mathcal{L}^{\perp}$. Let $j \in \mathbb{Z}$

$$
\begin{aligned}
\operatorname{Hom}_{\mathbf{D}(\mathcal{A})}\left(E[j], \mathrm{a} B_{0}\right) & =\operatorname{Hom}_{\mathbf{D}(\mathcal{A})}\left(\mathrm{ai} E[j], \mathrm{a} B_{0}\right) \\
& =\operatorname{Hom}_{\mathbf{D}(R)}\left(\mathrm{i} E[j], \mathrm{ia} B_{0}\right) \\
& \simeq \operatorname{Hom}_{\mathbf{D}(R)}\left(\mathrm{i} E[j], B_{0}\right) \\
& =0
\end{aligned}
$$

where the first equality holds because ai $E=E$, the third isomorphism because $\mathrm{ia} B_{0} \simeq B_{0}$ being $B_{0} \mathcal{L}_{\mathcal{A}}$-local, and the last equality because $B_{0}$ is $\mathcal{L}^{\prime}$-local.

Example Let $X$ be a noetherian separated scheme. Let $Z$ be a closed subscheme of $X$ and $i: X \backslash Z \rightarrow X$ the associated embedding. The functor $\mathcal{F} \mapsto i_{*} i^{*} \mathcal{F}$ defines an idempotent endofunctor in $\mathbf{D}\left(\mathcal{O}_{X^{-}} Q c o\right)$, namely $\mathbf{R} i_{*} i^{*} \mathcal{F}$. This is an example of a Bousfield localization that comes from right derivation from a localization in the corresponding abelian category. Not all localizations come up this way, though. Consider the functor defined by $\mathcal{F} \mapsto$ $\mathbf{R Q L} \Lambda_{Z} \mathcal{F}$. It is shown in [AJL, end of Remark $\left.(0.4)(\mathrm{a})\right]$, that it defines a localization in $\mathbf{D}\left(\mathcal{O}_{X}-Q c o\right)$ and it is clear that, in general, it does not come from a localization in $\mathcal{O}_{X}-Q c o$.

As a final application we show how to get Brown's representability theorem for $\mathbf{D}(\mathcal{A})$ with $\mathcal{A}$ a Grothendieck category, using our results and the fact that the theorem holds for $\mathbf{D}(R)$ as proved by Neeman. Denote by $\mathbf{A b}$ the category of abelian groups. Recall that a functor $\mathbf{T} \rightarrow \mathbf{A b}$ where $\mathbf{T}$ is a triangulated category is called homological if it takes triangles to exact sequences.

Theorem 5.8 Let $H: \mathbf{D}(\mathcal{A}) \rightarrow \mathbf{A b}^{\text {op }}$ be a (contravariant) homological functor that takes coproducts in $\mathbf{D}(\mathcal{A})$ to products in $\mathbf{A b}$. Then $H$ is representable.

Proof If $\mathcal{A}=R$-mod, the result follows from [N3, Theorem 3.1] as we have already remarked that $\mathbf{D}(R)$ is compactly generated. In general consider the composition:

$$
\mathbf{D}(R) \stackrel{\mathrm{a}}{\longrightarrow} \mathbf{D}(\mathcal{A}) \stackrel{H}{\longrightarrow} \mathbf{A b}^{\mathrm{op}} .
$$

The functor a preserves coproducts and is triangulated, so $H \circ \mathrm{a}$ is a homological functor and it is representable by the previous remark. Let $E \in \mathbf{D}(R)$ be the representing object, i.e., for every $Y \in \mathbf{D}(R),(H \circ \mathrm{a})(Y) \simeq \operatorname{Hom}_{\mathbf{D}(R)}(Y, E)$. The object $E$ is $\mathcal{L}_{\mathcal{A}}$-local. If $Y^{\prime} \in \mathcal{L}_{\mathcal{A}}$, then $\mathrm{a}\left(Y^{\prime}\right)=0$ and therefore, $\operatorname{Hom}_{\mathbf{D}(R)}\left(Y^{\prime}, E\right) \simeq(H \circ \mathrm{a})\left(Y^{\prime}\right)=0$. As a consequence, $\mathrm{ia}(E) \simeq E$.

Now we claim that the functor $H$ is represented by a $(E)$. Indeed, let $X \in \mathbf{D}(\mathcal{A})$, then $X=\mathrm{ai}(X)$ and we have the following chain of isomorphisms: 


$$
\begin{aligned}
\operatorname{Hom}_{\mathbf{D}(\mathcal{A})}(X, \mathrm{a} E) & =\operatorname{Hom}_{\mathbf{D}(\mathcal{A})}(\mathrm{ai} X, \mathrm{a} E) \\
& =\operatorname{Hom}_{\mathbf{D}(R)}(\mathrm{i} X, \mathrm{ia} E) \\
& \simeq \operatorname{Hom}_{\mathbf{D}(R)}(\mathrm{i} X, E) \\
& \simeq(H \circ \mathrm{a})(\mathrm{i} X) \\
& =H(X) .
\end{aligned}
$$

Corollary 5.9 Let $\mathbf{T}$ be a triangulated category (with "small hom-sets") and $F: \mathbf{D}(\mathcal{A}) \rightarrow \mathbf{T}$ a triangulated functor that preserves coproducts. Then $F$ has a right adjoint.

Proof The proof is formal and the same as [N3, Theorem 4.1].

\section{References}

[AJL] Leovigildo Alonso Tarrío, Ana Jeremías López and Joseph Lipman, Local homology and cohomology on schemes. Ann. Sci. École Norm. Sup. (4) 30(1997), 1-39.

[BBD] A. A. Beilinson, J. Bernstein and P. Deligne, Faisceaux pervers. Analysis and topology on singular spaces, I (Luminy, 1981), 5-171; Astérisque 100, Soc. Math. France, Paris, 1982.

[BN] Marcel Bökstedt and Amnon Neeman, Homotopy limits in triangulated categories. Compositio Math. (2) 86(1993), 209-234.

[Bo1] A. K. Bousfield, The localization of spectra with respect to homology. Topology (4) 18(1979), 257-281.

[Bo2] The Boolean algebra of spectra. Comment. Math. Helv. (3) 54(1979), 368-377.

[Bo2a] Correction to: "The Boolean algebra of spectra". Comment. Math. Helv. (4) 58(1983), 599-600.

[BK] A. K. Bousfield and D. M. Kan, Homotopy limits, completions and localizations. Lecture Notes in Math. 304, Springer-Verlag, Berlin-New York, 1972.

[CtHK] Jean-Louis Colliot-Thélène, Raymond T. Hoobler and Bruno Kahn, The Bloch-Ogus-Gabber theorem. In: Algebraic K-theory (Toronto, ON, 1996), Amer. Math. Soc., Providence, RI, 1998, 31-94.

[F] J. Franke, On the Brown representability theorem for triangulated categories. Preprint.

[GP] Nicolae Popesco and Pierre Gabriel, Caractérisation des catégories abéliennes avec générateurs et limites inductives exactes. C. R. Acad. Sci. Paris 258(1964), 4188-4190.

[I] Birger Iversen, Cohomology of sheaves. Universitext. Springer-Verlag, Berlin-New York, 1986.

[K] Bernhard Keller, Deriving DG categories. Ann. Sci. École Norm. Sup. (4) 27(1994), 63-102.

[L] Azriel Lévy, Basic set theory. Springer-Verlag, Berlin-New York, 1979.

[N1] Amnon Neeman, The connection between the K-theory localization theorem of Thomason, Trobaugh and Yao and the smashing subcategories of Bousfield and Ravenel. Ann. Sci. École Norm. Sup. (4) 25(1992), 547-566.

[N2] The chromatic tower for $D(R)$. Topology (3) 31(1992), 519-532.

[N3] The Grothendieck duality theorem via Bousfield's techniques and Brown representability. J. Amer. Math. Soc. (1) 9(1996), 205-236.

[N4] Triangulated categories. Book in preparation.

[P] Dieter Puppe, On the formal structure of stable homotopy theory. Colloq. algebr. Topology, Aarhus, 1962, $65-71$.

[Ri] Jeremy Rickard, Derived categories and stable equivalence. J. Pure Appl. Algebra (3) 61(1989), 303-317.

[Sp] N. Spaltenstein, Resolutions of unbounded complexes. Compositio Math. (2) 65(1988), 121-154.

[St] Bo Stenström, Rings of quotients. An introduction to methods of ring theory. Grundlehren Math. Wiss. 217. Springer-Verlag, Berlin-Heidelberg-New York, 1975. 
[Ve] Jean-Louis Verdier, Catégories dérivées. Quelques résultats (Etat 0). Sémin. Géom. algébr. Bois-Marie, SGA $4 \frac{1}{2}$, Lecture Notes in Math. 569(1977), 262-311.

[W] Charles A. Weibel, An introduction to homological algebra. Cambridge Stud. Adv. Math. 38. Cambridge University Press, Cambridge, 1994.

Departamento de Álxebra

Facultade de Matemáticas

Universidade de Santiago de Compostela

E-15771 Santiago de Compostela

Spain

email: lalonso@zmat.usc.es

Facultade de Informática

Campus de Elviña

Universidade da Coruña

E-15071 A Coruña

Spain

email:mariaj@udc.es
Departamento de Álxebra

Facultade de Matemáticas

Universidade de Santiago de Compostela

E-15771 Santiago de Compostela

Spain

email: jeremias@zmat.usc.es 